\title{
Prognostic Value of Vascular Invasion in Well-Differentiated Papillary Thyroid Carcinoma
}

\author{
Volkert B. Wreesmann, ${ }^{1}$ Iain J. Nixon, ${ }^{1}$ Michael Rivera, ${ }^{2}$ Nora Katabi, ${ }^{3}$ Frank Palmer, ${ }^{1}$ Ian Ganly, \\ Ashok R. Shaha, ${ }^{1}$ R. Michael Tuttle, ${ }^{4}$ Jatin P. Shah, ${ }^{1}$ Snehal G. Patel, ${ }^{1}$ and Ronald A. Ghossein ${ }^{3}$
}

Background: Vascular invasion (VI) is an important predictor of distant metastasis and possible radioactive iodine (RAI) benefit in follicular, Hürthle cell, and poorly differentiated thyroid carcinomas, but its role in welldifferentiated papillary thyroid cancer (WDTC) remains unclear.

Methods: Archived pathological material of all differentiated thyroid carcinoma patients undergoing primary surgical treatment at Memorial Sloan-Kettering Cancer Center between 1986 and 2003 was reviewed by two dedicated thyroid pathologists. Only WDTCs were included in the present study. Standard statistical methods were used to assess the relationship between VI and outcomes of interest, including 10-year disease-specific survival (DSS), regional recurrence-free survival (RRFS), and distant recurrence-free survival (DRFS).

Results: VI was present in 47 of 698 WDTC (6.7\%). VI was significantly associated with tumor size $>4.0 \mathrm{~cm}$, extrathyroidal extension, distant metastasis, and RAI treatment. On univariate analysis, VI was predictive of decreased 10-year DRFS, but not DSS or RRFS. On multivariate analysis, VI was not an independent predictor of DRFS. Univariate survival analysis of 422 RAI-naïve WDTC showed that both size $>4 \mathrm{~cm}$ and VI were predictors of outcome, but only size remained independently predictive on multivariate analysis.

Conclusion: The presence of VI is not an independent predictor of outcome in WDTC.

\section{Introduction}

W ELL-DIFFERENTIATED PAPILLARY THYROID CARCINOMA (PTC) accounts for $90 \%$ of thyroid cancers, and has a favorable cure rate $(95 \%)$, despite a significant risk for recurrence (up to 25\%) (1). Clinical management of PTC at our institution is guided by classification systems designed to predict survival such as GAMES (Grade, Age, Metastasis, Extrathyroidal extension, Size) and the American Joint Committee on Cancer's TNM, but also by those designed to predict recurrence such as the American Thyroid Association (ATA) system. However, these do not satisfactorily differentiate the small proportion of patients at risk for diseasespecific death and recurrence from the majority of innocuous PTC (2). As a result, most PTCs worldwide are treated aggressively with total thyroidectomy (with or without neck dissection) and adjuvant radioactive iodine (RAI) treatment, with the potential for significant morbidity (3). Despite a body of literature supporting de-intensified treatment for innocuous PTC, it is clear that such efforts will not succeed without delineation of a more accurate staging system (4-7). Modern thyroid pathology reporting includes a wide range of variables that were not directly included in the original staging systems but have significant potential to help decrease the uncertainty in considering an individual's level of risk.

Vascular invasion (VI), histologically defined by the presence of tumor cells within the lumen or walls of tumoral vessels and a reflection of an acquired propensity for lymphatic and hematogenous spread, is a controversial prognostic factor that has been included in the ATA recurrence risk prediction system. On the one hand, VI is associated with distant metastasis and putative benefit of systemic RAI treatment $(8,9)$ in follicular, Hürthle cell, and poorly differentiated thyroid tumors. On the other hand, the prognostic role of VI in PTC is unsatisfactorily supported by conflicting data from multiple studies (10-17), exposing the ATA recommendation to consider VI as a relative indicator of adjuvant RAI administration to significant debate (18).

As the current literature supporting VI as a trigger for aggressive therapy is limited by lack of pathological slide rereview, inclusion of heterogeneous study populations, and lack of multivariate analysis, the aim of the present study was to analyze the impact of VI on outcome in a large cohort of histologically confirmed PTC.

\footnotetext{
${ }^{1}$ Head and Neck Service, Department of Surgery; ${ }^{3}$ Department of Pathology; ${ }^{4}$ Endocrine Service, Department of Medicine; Memorial Sloan-Kettering Cancer Center, New York, New York.

${ }^{2}$ Department of Pathology, Mayo Clinic, Rochester, Minnesota.
} 


\section{Materials and Methods}

\section{Inclusion criteria}

All differentiated (non-anaplastic, non-medullary) thyroid carcinoma patients undergoing primary treatment at Memorial Sloan-Kettering Cancer Center between 1986 and 2003 were identified from the institutional database $(n=1282)$. All cases $(n=886)$ with available pathological slides were rereviewed by two dedicated thyroid pathologists (R.A.G. and M.R.). Patients without available pathological slides were excluded from the present study. Upon slide re-review, patients with follicular carcinoma, anaplastic carcinoma, poorly differentiated thyroid carcinoma, Hürthle cell carcinoma, and benign tumors (reclassified upon slide review using current pathological criteria) were excluded. Only patients with welldifferentiated PTC (Fig. 1 A and B) were included in the final analysis $(n=698)$.

\section{Pathological analysis}

Histopathologic review was performed by two dedicated thyroid pathologists who were blinded to the clinical characteristics and outcomes of the patients. VI was defined according to the criteria outlined in the Armed Forces Institute of Pathology fascicle (19). Briefly, for encapsulated tumors, VI was defined as invasion of a vessel within or outside the tumor capsule. If the tumor was not encapsulated, any VI inside or outside the tumor was considered as VI. The intravascular tumor growth had to be covered by endothelium or attached to the vessel wall or admixed with fibrin (Fig. 1C and D). Irregular clusters of non-endothelialized tumor cells that were not attached to the vessel wall represented artifactual dislodgement of tumor during sectioning and did not qualify as VI. The numbers of foci of VI were recorded. VI was defined as focal when fewer than four foci were detected, and extensive when four or more foci were detected, as described previously (9).

Pathologic criteria used to define the various thyroid tumor types are described below. A tumor was defined as classical PTC if it showed $>1 \%$ papillary formations, and was composed of cells having the characteristic nuclear features of papillary carcinoma (i.e., irregular enlarged clear nuclei with grooves and pseudo-inclusions; Fig. 1A and B). The classical PTC had to contain $<50 \%$ of tall cells. Tall-cell variant PTC was defined by the presence of $50 \%$ or more tall cells. Tall cells were defined as cells with their height twice their width and having an eosinophilic cytoplasm with a low nuclear/ cytoplasmic ratio and the characteristic nuclear features of PTC. Tumors were classified as follicular variant PTC (FVPTC) if they were composed of follicles lined by cells that had the typical characteristics of PTC described above, and contained $<1 \%$ well-formed papillae. FVPTC was further subdivided into non-encapsulated infiltrative when they displayed absent or incomplete encapsulation with invasive tongues of tumor infiltrating non-neoplastic thyroid parenchyma. FVPTC was defined as encapsulated when the tumor was completely surrounded by a tumor capsule with or without vascular or capsular invasion. Micro-PTC was defined as a PTC measuring $<1 \mathrm{~cm}$ in greatest size. All PTC that showed histologic evidence of poor differentiation defined by the presence of tumor necrosis and/or at least four mitoses per 10 high power fields $(\times 400)$ were excluded from the study. Follicular carcinomas, Hürthle cell tumors, anaplastic tumors, and benign tumors were diagnosed according to the criteria outlined in the World Health Organization monograph on endocrine tumors (20). The final study population contained 278 classical, 38 non-encapsulated FVPTC, 134 encapsulated FVPTC, 159 micro-PTC not otherwise specified, 81 tall-cell variant PTC, and eight rare variants (Table 1).
FIG. 1. Microphotographs of papillary thyroid carcinoma (PTC), classical type with vascular invasion (hematoxylin and eosin slides). (A) Low-power view of the carcinoma showing papillae (arrow). (B) On high power, the papillae are covered by cells with enlarged, clear, irregular, overlapping nuclei with grooves (arrow). This nuclear atypia is diagnostic of PTC. (C) Low-power view showing a tumor thrombus (TT) hanging in the lumen of a medium-sized extrathyroid vessel (delineated by arrows) located immediately outside the thyroid capsule. (D) High-power view of the tumor thrombus (TT) in (C) covered by endothelial cells (arrow).
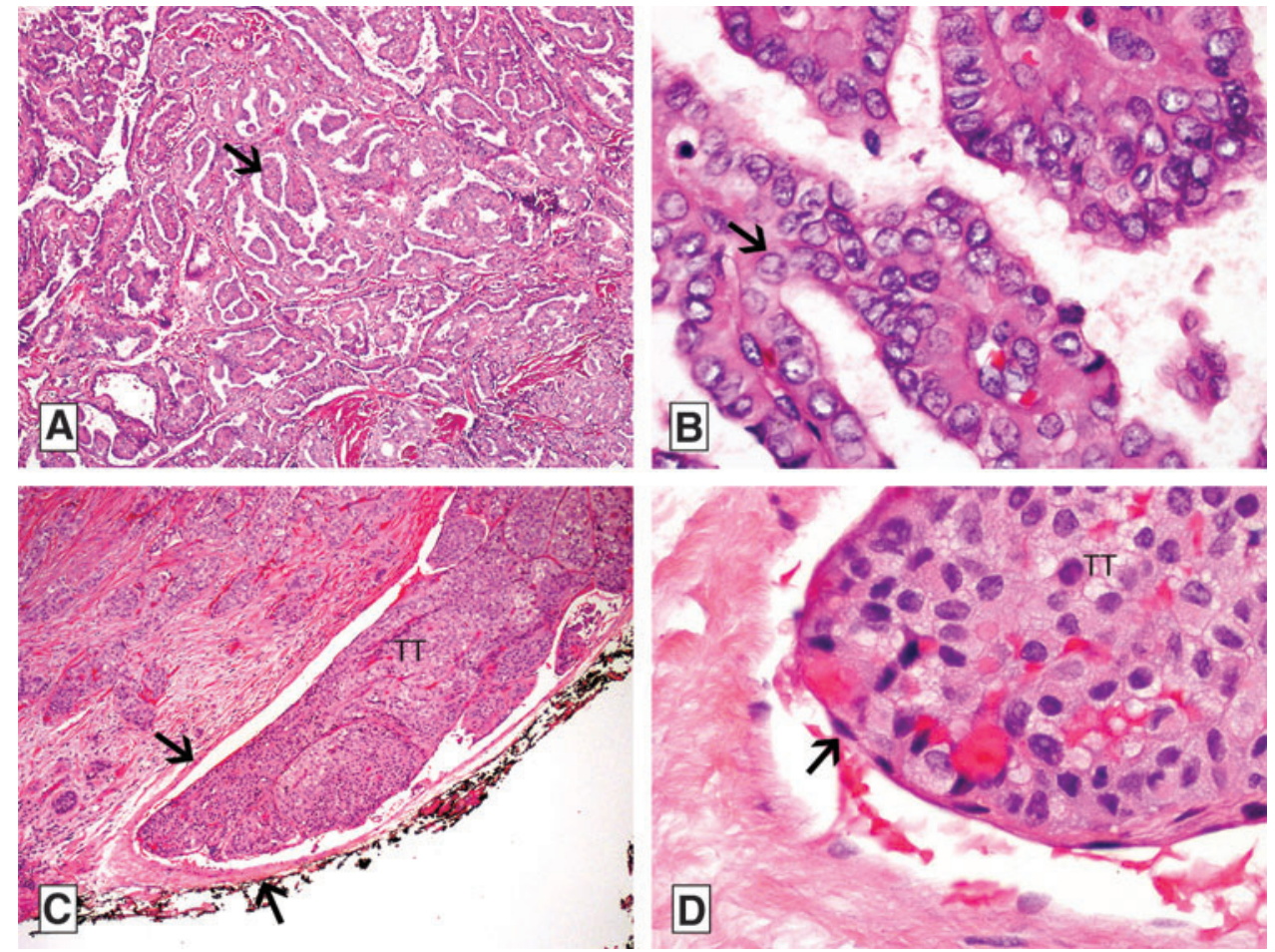
Table 1. Clinicopathological Profile of Study Population

\begin{tabular}{|c|c|c|}
\hline Variable & $\begin{array}{c}\text { No. of } \\
\text { patients }\end{array}$ & $\begin{array}{l}\% \text { of } \\
\text { total }\end{array}$ \\
\hline \multicolumn{3}{|l|}{ Age } \\
\hline$\leq 45$ & 348 & 49 \\
\hline$>45$ & 350 & 51 \\
\hline \multicolumn{3}{|l|}{ Sex } \\
\hline Female & 505 & 72 \\
\hline Male & 193 & 28 \\
\hline \multicolumn{3}{|l|}{ Tumor size } \\
\hline$\leq 4 \mathrm{~cm}$ & 606 & 87 \\
\hline$>4 \mathrm{~cm}$ & 92 & 13 \\
\hline \multicolumn{3}{|l|}{ Gross extrathyroidal extension } \\
\hline Present & 78 & 11 \\
\hline Absent & 620 & 89 \\
\hline \multicolumn{3}{|l|}{ Margins } \\
\hline Negative & 619 & 89 \\
\hline Positive & 79 & 11 \\
\hline \multicolumn{3}{|l|}{ Nodal metastasis } \\
\hline$<4$ nodes & 554 & 79 \\
\hline$\geq 4$ nodes & 144 & 21 \\
\hline \multicolumn{3}{|l|}{ Distant metastasis (at presentation) } \\
\hline Present & 16 & 3 \\
\hline Absent & 682 & 97 \\
\hline \multicolumn{3}{|l|}{ Stage } \\
\hline I & 499 & 71 \\
\hline II & 39 & 6 \\
\hline III & 82 & 12 \\
\hline IV & 78 & 11 \\
\hline \multicolumn{3}{|l|}{ Thyroid surgery } \\
\hline Total thyroidectomy & 473 & 68 \\
\hline Less than total thyroidectomy & 225 & 32 \\
\hline \multicolumn{3}{|l|}{ Neck dissection } \\
\hline Central & 113 & 16 \\
\hline Lateral & 43 & 6 \\
\hline Central and lateral & 114 & 16 \\
\hline \multicolumn{3}{|l|}{ RAI } \\
\hline Yes & 276 & 40 \\
\hline No & 422 & 60 \\
\hline \multicolumn{3}{|l|}{ Histological subtype } \\
\hline Classical PTC & 278 & 40 \\
\hline $\begin{array}{l}\text { Non-encapsulated follicular } \\
\text { variant PTC }\end{array}$ & 38 & 5 \\
\hline $\begin{array}{l}\text { Encapsulated follicular } \\
\text { variant PTC }\end{array}$ & 134 & 19 \\
\hline Tall-cell variant PTC & 81 & 12 \\
\hline Micro-PTC & 159 & 23 \\
\hline Miscellaneous variants of PTC & 8 & 1 \\
\hline
\end{tabular}

RAI, radioactive iodine; PTC, papillary thyroid carcinoma.

\section{Statistical analysis}

The primary endpoints of interest included 10-year diseasespecific survival (DSS), regional recurrence-free survival (RRFS), and distant recurrence-free survival (DRFS). Recurrence included structural disease recurrence defined as biopsy proven or structural abnormalities on imaging that were deemed highly suspicious of thyroid cancer recurrence. Survival outcomes were calculated by the Kaplan-Meier method; groups were compared with univariate analysis using the log-rank test. A $p$-value of $\leq 0.05$ was considered statistically significant. Prognostic factors that were significant on univariate analysis were analyzed in multivariate Cox proportional hazards models for independent significance. Nonparametric qualitative and quantitative comparisons were performed using Fisher's exact or Pearson's chi-square tests and the Mann-Whitney $U$-test, respectively.

\section{Results \\ Clinical data}

All clinical data of patients selected for the current study based on pathologic re-review were transferred from the institutional database to a separate database for analysis. The clinicopathological profile of the study population is summarized in Table 1 . The median age of the study population was 45 years (range 1-87 years). Out of 698 patients, 505 $(72 \%)$ were female. The median primary tumor size was $1.5 \mathrm{~cm}$ (range $0.04-9 \mathrm{~cm})$. In 606 patients $(87 \%)$, the primary tumor size was $<4 \mathrm{~cm}$. Extrathyroidal extension was present in 193 patients (28\%), of which 115 manifested microscopic extrathyroidal extension, and 78 patients gross extrathyroidal extension. Distant metastasis at presentation was recorded in 16 patients $(3 \%)$. In 436 patients, no clinical/radiological evidence of regional metastasis was obtained at presentation. Primary treatment consisted of total thyroidectomy in 473 cases $(68 \%)$, lobectomy in 215 patients $(31 \%)$, and isthmusectomy in 10 patients $(1.4 \%)$. Lymph node dissection consisted of central neck dissection in 227 patients (32\%), and lateral neck dissection in 157 patients $(22 \%)$. More than three positive lymph nodes were present in 144 patients $(21 \%)$. RAI was delivered to 276 patients (40\%). The median followup was 81 months (range 1-320 months).

\section{$\mathrm{VI}$ and prognostic factors}

Slide review of 698 well-differentiated PTC identified the presence of VI in 47 cases $(6.7 \%)$. The median number of identified VI foci was 1 (range 1-11). VI was defined as focal in 35 cases, and extensive VI in 12 cases. Extrathyroidal VI was present in 18 cases only. The association of VI to various patient, tumor, and treatment factors is described in Table 2. The presence of VI was significantly associated with poor prognostic factors, including tumor size $>4 \mathrm{~cm}$ (5\% vs. $20 \%$; $p<0.0001)$, gross extrathyroidal extension (6\% vs. $15 \%$; $p<0.001)$, and distant metastasis (6\% vs. $25 \% ; p=0.001)$. VI was also significantly associated with RAI treatment

TABLE 2. RELATIONSHIP OF VI with Clinicopathological Factors

\begin{tabular}{lrlc}
\hline Factor & \multicolumn{1}{c}{ No VI } & VI & p-Value \\
\hline Size $\leq 4 \mathrm{~cm}$ & $598(95 \%)$ & $35(5 \%)$ & \\
Size > cm & $43(80 \%)$ & $11(20 \%)$ & 0.000 \\
No ETE & $585(94 \%)$ & $35(6 \%)$ & \\
Gross ETE & $66(85 \%)$ & $12(15 \%)$ & 0.001 \\
Age $\leq 45$ years & $319(92 \%)$ & $29(8 \%)$ & \\
Age > 45 years & $332(95 \%)$ & $18(5 \%)$ & 0.09 \\
No DM & $639(94 \%)$ & $43(6 \%)$ & \\
DM & $12(75 \%)$ & $4(25 \%)$ & 0.001 \\
No RAI & $407(95 \%)$ & $21(5 \%)$ & \\
RAI & $250(91 \%)$ & $26(9 \%)$ & 0.022 \\
\hline
\end{tabular}

VI, vascular invasion; ETE, extrathyroidal extension; DM, distant metastases. 


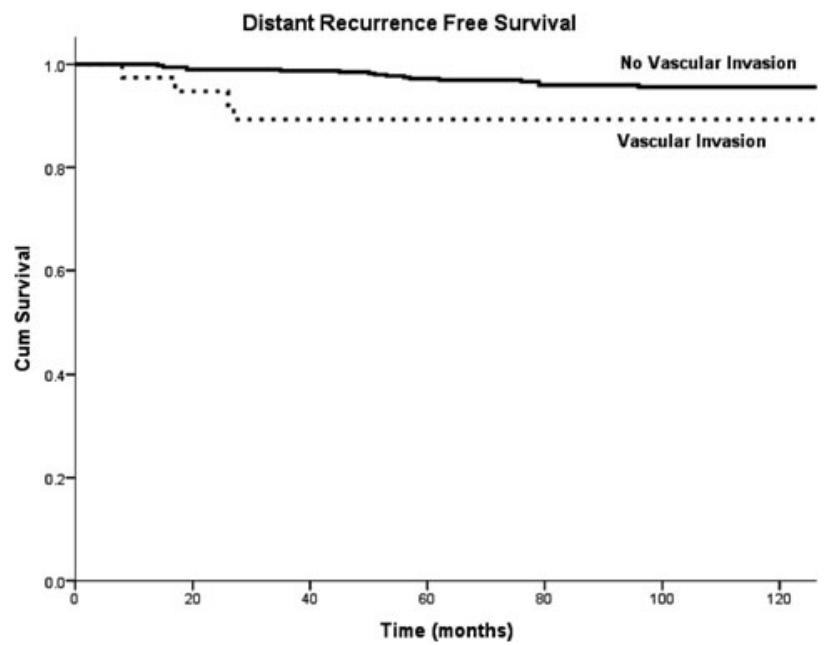

FIG. 2. Univariate Kaplan-Meyer analysis of distant recurrence-free survival (DRFS) of patients with and without vascular invasion (VI). Patients with vascular invasion have a significantly decreased DRFS compared to patients without VI.

( $5 \%$ vs. $10 \% ; p=0.022)$. Location or extent of VI was not associated with any of the clinicopathological factors.

\section{VI and outcome}

Univariate survival analysis as a function of VI and established prognostic factors was performed. The presence of VI was associated with decreased DRFS (95.6\% vs. $89.3 \%$; $p=0.031$; Fig. 2). VI was not associated with RRFS $(93.5 \%$ vs. $88.5 \% ; p=0.727)$ or DSS $(97.3 \%$ vs. $97.2 \% ; p=0.964)$. The extent of VI (focal vs. extensive) and its location (intravs. extrathyroidal) did not have a significant impact on survival. Beside VI, other significant predictors of DRFS included tumor size $>4 \mathrm{~cm}(p<0.001)$, gross ETE $(p<$ $0.001)$, RAI administration $(p<0.001)$, and age $>45$ years $(p=0.025)$. Because of the limited number of distant recurrence events $(n=22)$, it was not possible to enter all significant univariate predictors of DRFS simultaneously into one model. Instead, a sequential bivariate analysis was performed, in which each significant variable was analyzed individually in a model together with VI. In none of these bivariate analyses was VI an independent prognostic factor.

\section{VI in RAl-naïve PTC}

To assess the impact of VI on DRFS independent of the effect of RAI treatment on clinical outcomes, a subset analysis was performed of the 422 RAI-naïve PTC within our study population. VI was identified in 21 of 422 cases (4.9\%). Table 3 describes the assessment of putative prognostic factors of DRFS in RAI-native PTC. Both the presence of VI and tumor size $>4 \mathrm{~cm}$ were significant poor prognostic factors on univariate analysis. In multivariate analysis, only tumor size remained an independent predictor of DRFS $(p=0.003)$.

\section{Discussion}

Although the prognostic importance of VI is well characterized in follicular, Hürthle cell, and poorly differentiated
TABle 3. Univariate DRFS Analysis IN RAI-NAÏVE PTC $(N=422)$

\begin{tabular}{lcc}
\hline Factor $(\mathrm{n})$ & $\begin{array}{c}\text { 10-year distant RFS } \\
\text { (three events) }\end{array}$ & p-Value \\
\hline No VI (401) & $99.6 \%$ & \\
VI (21) & $93.3 \%$ & 0.013 \\
ETE & $100 \%$ & 0.669 \\
No ETE & $99.2 \%$ & 0.455 \\
Age $\leq 45$ years & $99.3 \%$ & \\
Age $>45$ years & $99.1 \%$ & \\
No DM & $100 \%$ & 0.000 \\
DM & - & \\
Size $\leq 4 \mathrm{~cm}$ & $100 \%$ & \\
Size $>4 \mathrm{~cm}$ & $83.6 \%$ &
\end{tabular}

DRFS, distant recurrence-free survival.

thyroid cancer, its influence on outcome in PTC is controversial. First, previous studies (10-17) demonstrate significant variability in the incidence of VI in PTC ranging from $5.6 \%$ to $33 \%$. Second, significant variability with respect to the relationship between VI and outcome exists among prior studies. Although recurrence rates were found to be significantly higher when VI was present in the studies by Gardner et al. (16-20\% with VI, 3-6\% without), Nishida et al. (28\% with VI, $15 \%$ without), and Falvo et al. (30\% with VI, 5\% without) $(11,14,16)$, studies by Furlan et al. and Akslen et al. failed to confirm this observation $(15,17)$. Similarly, VI in PTC was associated with higher rates of distant metastases $(12,13,16)$ and disease-specific mortality $(10,17)$ in some but not all studies. The variability between studies may be related to interobserver variability pertaining to the histologic identification of VI, lack of pathological slide re-review, inclusion of follicular and poorly differentiated thyroid tumors, and lack of multivariate analysis. Conflicting data from prior studies leave the administration of adjuvant RAI in the setting of VI subject to ongoing debate.

This study presents the largest series of patients with PTC reviewed with regards to VI. The data suggest that the presence of VI is an indicator of decreased DRFS, but that this effect is not independent from established outcome predictors in PTC, even in patients without a history of systemic postoperative treatment with RAI. As the presence of VI is strongly associated with established poor prognostic factors of WDTC, the clinical significance of cases with VI in the absence of these factors remains to be ascertained. In the present series, the outcome of all VI positive patients with absence of extrathyroidal extension, negative surgical margins, fewer than three ascertained positive regional metastases, and tumor size $<4 \mathrm{~cm}$, without a history of RAI treatment $(n=12)$ was assessed. It is of note that none of these patients had any detectable disease-specific adverse event at a median follow-up of 95 months (data not shown). Outcome was not influenced by the extent of VI, with no significant differences in outcome between extensive and focal VI in the study. This is in contrast to what was observed previously in encapsulated Hürthle cell carcinomas (21), and could be due to the small number of patients with extensive VI $(n=12)$.

An important strength of this study includes the inclusion of a large cohort of homogeneous cases $(n=1282)$ with significant follow-up (median follow-up 10 years). For the 
purpose of this study, all slides were carefully reviewed by two expert thyroid pathologists, leading to inclusion of patients with well-differentiated PTC by contemporary histopathological standards only. This is important, given the complex histological phenotype of thyroid cancers, the continuous evolution of diagnostic criteria in thyroid cancer, and the high rate of interobserver variability in the diagnosis of certain subtypes of PTC, even among expert endocrine pathologists (22). Inclusion of a large number of patients through slide review also ensured that outcome analysis could be assessed based on a relatively large number of VI events, and criteria for determination of VI were uniform. The present study is by far the largest compared to prior studies, has the longest follow-up, and is one of the few thyroid cancer studies that is based on pathological slide re-review.

Despite these strengths, interpretation of the present data is limited by its retrospective nature. A potential caveat for bias includes the fact that patients without available pathological slides were excluded from the present analysis. This influence may have skewed the analysis of the present study toward inclusion of patients from more recent years. Although a comparison of prognostic factors and outcomes between the groups of patients with and without available slides did not demonstrate significant differences (results not shown), the possibility of bias that may have arisen from this issue cannot be ruled out. Another concern that may arise during interpretation of the study involves the possibility that RAI administration influenced the absence of independent predictive significance of VI on outcome parameters in the overall group of patients, by resolving micrometastatic PTC deposits that may have led to adverse outcome events if no RAI had been applied. Although a separate analysis was performed including only patients without a history of RAI treatment, this analysis is clearly affected by selection bias, given the divergent profile of prognostic factors between the RAI-naive data set and the overall data set.

Altogether, it is concluded that VI in well-differentiated PTC typically occurs in concert with other clinicopathological indicators of aggressive behavior that may together guide a decision for RAI administration. In cases of welldifferentiated PTC with VI and absence of other aggressive features, increased risk of recurrence or death is not suggested by the data. It remains to be confirmed whether such cases can safely be managed without RAI treatment.

\section{Author Disclosure Statement}

No competing conflicts of interest to disclose for any of the authors.

\section{References}

1. Shaha AR 2012 Recurrent differentiated thyroid cancer. Endocr Pract 18:600-603.

2. Momesso DP, Tuttle RM 2014 Update on Differentiated Thyroid Cancer Staging. Endocrinol Metab Clin North Am 43:401-421.

3. Guerrero MA, Clark OH 2011 Controversies in the management of papillary thyroid cancer revisited. ISRN Oncol 2011:303128.
4. Ibrahimpasic T, Nixon IJ, Palmer FL, Whitcher MM, Tuttle RM, Shaha A, Patel SG, Shah JP, Ganly I 2012 Undetectable thyroglobulin after total thyroidectomy in patients with low- and intermediate-risk papillary thyroid cancer-is there a need for radioactive iodine therapy? Surgery 152:1096-1105.

5. Nixon IJ, Ganly I, Patel SG, Palmer FL, Whitcher MM, Tuttle RM, Shaha A, Shah JP 2012 Thyroid lobectomy for treatment of well differentiated intrathyroid malignancy. Surgery 151:571-579.

6. Vaisman F, Momesso D, Bulzico DA, Pessoa CH, da Cruz MD, Dias F, Corbo R, Vaisman M, Tuttle RM 2013 Thyroid lobectomy is associated with excellent clinical outcomes in properly selected differentiated thyroid cancer patients with primary tumors greater than $1 \mathrm{~cm}$. J Thyroid Res 2013:398194.

7. Tuttle RM, Sabra MM 2013 Selective use of RAI for ablation and adjuvant therapy after total thyroidectomy for differentiated thyroid cancer: a practical approach to clinical decision making. Oral Oncol 49:676-683.

8. Ghossein R 2009 Problems and controversies in the histopathology of thyroid carcinomas of follicular cell origin. Arch Pathol Lab Med 133:683-691.

9. Stojadinovic A, Ghossein RA, Hoos A, Urist MJ, Spiro RH, Shah JP, Brennan MF, Shaha AR, Singh B 2001 Hürthle cell carcinoma: a critical histopathologic appraisal. J Clin Oncol 19:2616-2625.

10. Simpson WJ, McKinney SE, Carruthers JS, Gospodarowicz MK, Sutcliffe SB, Panzarella T 1987 Papillary and follicular thyroid cancer. Prognostic factors in 1,578 patients. Am J Med 83:479-488.

11. Nishida T, Katayama S, Tsujimoto M 2002 The clinicopathological significance of histologic vascular invasion in differentiated thyroid carcinoma. Am J Surg 183:80-86.

12. Mete O, Asa SL 2011 Pathological definition and clinical significance of vascular invasion in thyroid carcinomas of follicular epithelial derivation. Mod Pathol 24:1545-1552.

13. Mai KT, Khanna P, Yazdi HM, Perkins DG, Veinot JP, Thomas J, Lamba M, Nair BD 2002 Differentiated thyroid carcinomas with vascular invasion: a comparative study of follicular, Hürthle cell and papillary thyroid carcinoma. Pathology 34:239-244.

14. Gardner RE, Tuttle RM, Burman KD, Haddady S, Truman C, Sparling YH, Wartofsky L, Sessions RB, Ringel MD 2000 Prognostic importance of vascular invasion in papillary thyroid carcinoma. Arch Otolaryngol Head Neck Surg 126:309-312.

15. Furlan JC, Bedard YC, Rosen IB 2004 Clinicopathologic significance of histologic vascular invasion in papillary and follicular thyroid carcinomas. J Am Coll Surg 198:341348.

16. Falvo L, Catania A, D’Andrea V, Marzullo A, Giustiniani MC, De Antoni E 2005 Prognostic importance of histologic vascular invasion in papillary thyroid carcinoma. Ann Surg 241:640-646.

17. Akslen LA, Myking AO, Salvesen H, Varhaug JE 1992 Prognostic importance of various clinicopathological features in papillary thyroid carcinoma. Eur J Cancer 29A: 44-51.

18. American Thyroid Association Guidelines Taskforce on Thyroid Nodules and Differentiated Thyroid Cancer, Cooper DS, Doherty GM, Haugen BR, Kloos RT, Lee SL, Mandel SJ, Mazzaferri EL, McIver B, Pacini F, Schlumberger M, Sherman SI, Steward DL, Tuttle RM 2009 Revised American Thyroid 
Association management guidelines for patients with thyroid nodules and differentiated thyroid cancer. Thyroid 19:1167-1214.

19. Rosai J Carcangiu ML, De Lellis RA 1992 Tumors of the Thyroid Gland. Armed Forces Institute of Pathology, Washington, DC.

20. DeLellis RA Lloyd RV, Heitz PU, Eng C, eds 2004 World Health Organization Classification of Tumours. Pathology and Genetics Tumours of the Endocrine Organs. IARC Press, Lyon.

21. Ghossein RA, Hiltzik DH, Carlson DL, Patel S, Shaha A, Shah JP, Tuttle RM, Singh B 2006 Prognostic factors of recurrence in encapsulated Hürthle cell carcinoma of the thyroid gland: a clinicopathologic study of 50 cases. Cancer 106:1669-1676.
22. Lloyd RV, Erickson LA, Casey MB, Lam KY, Lohse CM, Asa SL, Chan JK, DeLellis RA, Harach HR, Kakudo K, LiVolsi VA, Rosai J, Sebo TJ, Sobrinho-Simoes M, Wenig BM, Lae ME 2004 Observer variation in the diagnosis of follicular variant of papillary thyroid carcinoma. Am J Surg Pathol 28:1336-1340.

Address correspondence to: Ronald Ghossein, MD Department of Pathology Memorial Sloan-Kettering Cancer Center 1275 York Avenue New York, NY 10065

E-mail: ghosseir@mskcc.org 